\title{
Suppression of spin beats in magneto-oscillation phenomena in two-dimensional electron gas
}

\author{
N.S. Averkiev, M.M. Glazov, and S.A. Tarasenko \\ A.F. Ioffe Physico-Technical Institute of the RAS, 194021 St. Petersburg, Russia
}

\begin{abstract}
Theory of magneto-oscillation phenomena has been developed for two-dimensional electron systems with linear-in- $\boldsymbol{k}$ spin splitting. Both Dresselhaus and Rashba contributions are taken into account. It has been shown that the pattern of the magneto-oscillations depends drastically on the ratio between the above terms. The presence of only one type of the $\boldsymbol{k}$-linear terms gives rise to the beats, i.e. two close harmonics corresponding to the spin-split subbands. However, if the strengths of both contributions are comparable, the third (central) harmonics appears in the spectrum of the magneto-oscillations. For equal strengths of the contributions, only the central harmonic survives, and the oscillations occur at a single frequency, although the $\boldsymbol{k}$-linear terms remain in the Hamiltonian. Such suppression of the spin beats is studied in detail by the example of the Shubnikov-de Haas effect.
\end{abstract}

\section{INTRODUCTION}

Spin-dependent transport phenomena in two-dimensional electron systems is of broad interest at present time. The peculiar property of the two-dimensional systems based on quantum wells is linear in the wave vector $\boldsymbol{k}$ spin-dependent terms in the effective Hamiltonian [1, 2]. These terms are caused by spin-orbit interaction that couples spin states and space motion of conduction electrons and governs the wide class of spin phenomena. Among them are spin relaxation, spin transport controlled with an external electric field by the Rashba effect, circular photogalvanic and spin-galvanic effects, electric current-induced spin orientation and precession, intrinsic spin Hall effect, etc.

Experimentally one of the most efficient methods for determination of the spin splitting in 2D conducting structures is measurements of oscillations of the magnetoresistivity (Shubnikov-de Haas effect). The quantum oscillations are highly sensitive to the fine structure of the energy spectrum of carriers, so that even small spin splitting, small as compared to the Fermi energy but commensurable with the energy distance between Landau levels, qualitatively modifies the oscillation behavior. The linear in the wave vector terms in the effective Hamiltonian remove the degeneracy in the carrier spectrum. In a magnetic field, the spin splitting at the Fermi surface gives rise to the oscillations with close frequencies, i.e. to beats 1]. Such a behavior was observed and attributed to the zero-field spin splitting in 2D electron gas under study of the Shubnikov-de Haas oscillations in InAs/GaSb 3 , 4], InGaAs/InAlAs [5, 6, 7], InGaAs/InP [8], AlGaAs/GaAs [9], InAs/AlSb 10], and InGaN/GaNbased 11] structures, and microwave radiation-induced magnetoresistance oscillations in GaAs/AlGaAs heterostructures [12]. The analysis of the oscillations was applied for determination of the spin splitting at the Fermi level. However, recently it was pointed out that the simple analysis of the beating pattern may lead to an incorrect conclusion on the spin splitting [13, 14]. In particularly, it was shown that the $\boldsymbol{k}$-linear contribution in- duced by heteropotential asymmetry (Rashba term) and the contribution originated from the cubic terms of a bulk material lacking inversion center (Dresselhaus term) interfere in the magneto-oscillation phenomena [14]. The presence of only one type of the linear terms gives rise to the beats. However, if the strengths of both contributions are equal, the oscillations occur only at a single frequency and the beats disappear, although the $\boldsymbol{k}$-linear terms remain in the Hamiltonian.

In this communication we present a theory of magnetooscillation phenomena in two-dimensional electron gas in the presence of $\boldsymbol{k}$-linear spin splitting. Both $\boldsymbol{k}$-linear contributions originated form a heteropotential asymmetry and the lack of the inversion center in a bulk semiconductor are taken into account. We take the Shubnikov-de Haas effect as an example to study the suppression of the spin beats in detail. The magnetoresistance is calculated for arbitrary ratio between the Rashba and Dresselhaus contributions. The Zeeman splitting of electronic levels is neglected for simplicity, since it is small compared to the spacing between the Landau levels in the magnetic field perpendicular to the quantum well plane in the majority of semiconductor structures based on the III-V compounds.

\section{THEORY}

Generally, appearance of the $\boldsymbol{k}$-linear terms is connected with reduction of the system symmetry as compared to the bulk material. In (001)-grown quantum wells based on zinc-blende-lattice semiconductors, there are two types of the linear contributions to the effective Hamiltonian of 2D electrons. First, they originate from the lack of the inversion center in the bulk material (BIA term) 2] (or from the asymmetry of the chemical bonds at interfaces (IIA term) 15]). This so-called Dresselhaus contribution has the form

$$
\mathcal{H}_{D}=\alpha\left(\sigma_{x} k_{y}+\sigma_{y} k_{x}\right),
$$

where $\sigma_{i}(i=x, y)$ are the Pauli matrices and the axes $x$ and $y$ are assumed to be parallel to the crystallographic 
axes [11̄0] and [110], respectively. Second, a linear contribution can be induced by the heterostructure asymmetry unrelated to the crystal lattice (SIA or Rashba term [1])

$$
\mathcal{H}_{R}=\beta\left(\sigma_{x} k_{y}-\sigma_{y} k_{x}\right) .
$$

Experimental data evidence that the intensities of the Dresselhaus and Rashba terms can be comparable in real 2D structures based on quantum wells [16, 17, 18]. Furthermore, the relative intensities of the contributions can be controlled tuning the heteropotential asymmetry with an external electric field normal to the quantum well plane.

In two-dimensional systems small magneto-oscillations occur in moderate magnetic fields, when $\omega_{c} \tau \leq 1$, where $\omega_{c}=e B / m^{*} c$ is the cyclotron frequency, $\tau$ is the scattering time, $e$ is the elementary charge, $\boldsymbol{B}$ is the applied magnetic field, $m^{*}$ is the effective electron mass, and $c$ is the light velocity. The corresponding parameter that determines the amplitude of the oscillations appears to be $\exp \left(-\pi / \omega_{c} \tau\right)[19,20,21,22]$. We assume that the inequality $E_{F} \tau / \hbar \gg 1$ providing good conductivity is fulfilled, and the spin splitting at the Fermi surface is much less than the Fermi energy $E_{F}$ but exceeds the spacing between the Landau levels, $\hbar \omega_{c} \ll \sqrt{\alpha^{2}+\beta^{2}} k_{F} \ll E_{F}$, where $k_{F}$ is the Fermi wave vector. Then, in the selfconsistent Born approximation, the single-particle electron Green's function under electron scattering by shortrange defects has the form

$$
\hat{\mathcal{G}}_{\varepsilon}\left(\boldsymbol{r}, \boldsymbol{r}^{\prime}\right)=\sum_{n, k_{y}} \frac{\Psi_{n k_{y}}(\boldsymbol{r}) \Psi_{n k_{y}}^{\dagger}\left(\boldsymbol{r}^{\prime}\right)}{\varepsilon+E_{F}-E_{n}-X_{\varepsilon}},
$$

where $\Psi_{n k_{y}}(\boldsymbol{r})$ are the spinor wave functions for an electron subjected to the magnetic field $\mathbf{B} \| z$ with the vector potential taken in the Landau gauge, $\boldsymbol{A}=(0, B x, 0), E_{n}$ are the electron levels, $X_{\varepsilon}$ is the self-energy part of the Green's function, $k_{y}$ is the $y$-component of the wave vector, and $n$ is the quantum number enumerating both the Landau levels and the spin states. We note, that the Green's function (3) is a $2 \times 2$ matrix in the spin indices. The self-energy part obeys, for the large numbers $n \sim 2 E_{F} / \hbar \omega_{c}$, the following equation

$$
X_{\varepsilon}=\frac{\hbar^{2} \omega_{c}}{4 \pi \tau} \sum_{n} \frac{1}{\varepsilon+E_{F}-E_{n}-X_{\varepsilon}} .
$$

Quantum oscillations are determined, to the first order in the parameter $\exp \left(-\pi / \omega_{c} \tau\right)$, by the imaginary part of the self-energy. The real part of $X_{\varepsilon}$ can be assumed to be included in the Fermi energy $E_{F}$. Within this accuracy the solution of the Eq. (4) has the form

$$
X_{\varepsilon}=-i \frac{\hbar^{3} \omega_{c}}{8 \pi \tau} \sum_{n} \frac{\operatorname{sign} \varepsilon}{\left(\varepsilon+E_{F}-E_{n}\right)^{2}+(\hbar / 2 \tau)^{2}} .
$$

The eigen wave functions $\Psi_{n k_{y}}(\boldsymbol{r})$ and the energies $E_{n}$ are determined from the solution of the Schrödinger equation, with the terms $\mathcal{H}_{\mathrm{D}}$ and $\mathcal{H}_{\mathrm{R}}$ being included in the
Hamiltonian. In the presence of only one type of the linear terms (either Dresselhaus or Rashba), electron states in the magnetic field can be conveniently characterized by two indices $n=(m, s)$, where the index $s= \pm$ denotes spin states. Then the electron energies are given by [1]

$$
E_{m, \pm}=\hbar \omega_{c} m \pm \sqrt{4 \alpha^{2} m / \lambda_{B}^{2}+\left(\hbar \omega_{c}\right)^{2} / 4}
$$

where $\lambda_{B}=\sqrt{\hbar c / e B}$ is the magnetic length. It is the splitting $E_{m,+}-E_{m,-}=4 \alpha \sqrt{m} / \lambda_{B} \approx 2 \alpha k_{F}$ that gives rise to the beats in magneto-oscillations [1, 14, 23, 24]. In the other limiting case, $|\alpha|=|\beta|$, the orbital motion and the spin states can be separated, and the electron levels become double-fold degenerate 14

$$
E_{m, \pm}=\hbar \omega_{c}(m+1 / 2)-2 m^{*} \alpha^{2} / \hbar^{2} .
$$

The $\boldsymbol{k}$-linear terms, although being present in the Hamiltonian, do not lead to the splitting of the Landau levels, and the beats in the magneto-oscillations do not occur.

For the arbitrary strengths of the Dresselhaus and Rashba terms the electron spectrum in the magnetic field can be calculated numerically. Fig. 1 presents the dependence of the arrangement of the Landau levels on the ratio $\beta / \alpha$ calculated for the value $m^{*} \alpha^{2} /\left(2 \omega_{c} \hbar^{3}\right)=0.045$ that is an estimation for $\mathrm{GaAs} / \mathrm{AlGaAs}$ structures in the magnetic field $1 \mathrm{~T}$. One can see that the dependence of the energy spectrum on $\beta / \alpha$ is rather complicated. At arbitrary ratio $\beta / \alpha$ the electron states are nondegenerate, and one can expect the complicated pattern of the Shubnikov-de Haas oscillations. However, when $\alpha=\beta$ the spectrum is a set of the equidistant Landau levels with the energy distance $\hbar \omega_{c}$, and the oscillations occur at a single frequency.

The Green's function allows us to calculate various kinetic and thermodynamic coefficients. We demonstrate it taking the Shubnikov-de Haas effect as an example.

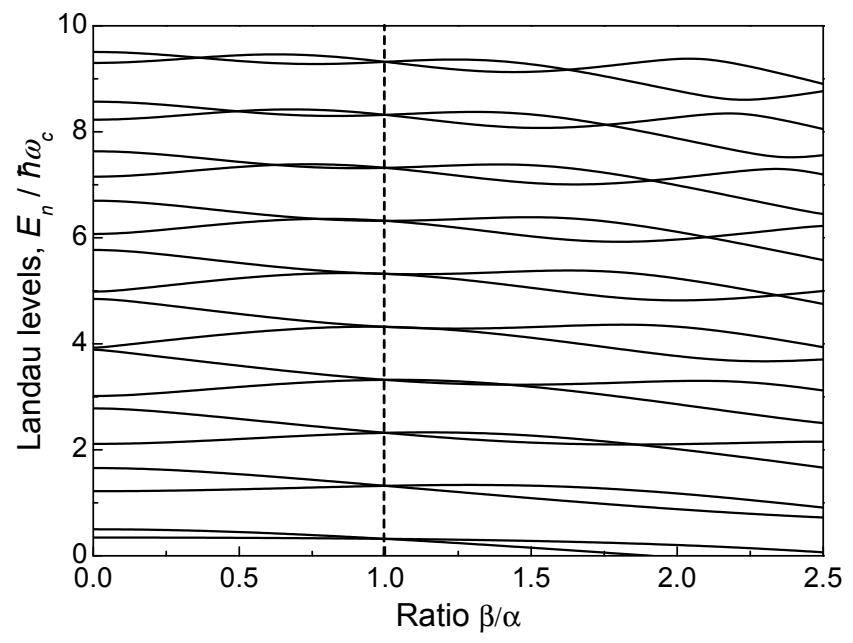

FIG. 1: The positions of the Landau levels at different ratio $\beta / \alpha$ for the fixed magnetic field and $m^{*} \alpha^{2} /\left(2 \omega_{c} \hbar^{3}\right)=0.045$. 
The conductivity tensor at a frequency of the external electric field $\omega$ is expressed in the terms of the Green's function by

$$
\begin{aligned}
& \sigma_{i j}(\omega)=\mathrm{i} \frac{N e^{2}}{m^{*} \omega} \delta_{i j}+\frac{1}{\omega} \operatorname{Tr} \iint d \boldsymbol{r} d \boldsymbol{r}^{\prime} \\
& \times \int_{-\infty}^{\infty} \frac{d \varepsilon}{2 \pi}\left[\hat{J}_{i}(\boldsymbol{r}) \hat{\mathcal{G}}_{\varepsilon+\hbar \omega}\left(\boldsymbol{r}, \boldsymbol{r}^{\prime}\right)\right]\left[\hat{J}_{j}\left(\boldsymbol{r}^{\prime}\right) \hat{\mathcal{G}}_{\varepsilon}\left(\boldsymbol{r}^{\prime}, \boldsymbol{r}\right)\right] .
\end{aligned}
$$

Here $N$ is the carrier concentration, $\delta_{i j}$ is the Kroneker symbol, $\hat{J}_{i}$ is the current density operator that, in general case, is a $2 \times 2$ matrix in the spin indices, $i, j=x, y$ are the in-plane co-ordinates, and the trace Tr implies summation over the spin indices. Below we are interested in the static conductivity, and therefore consider the frequency $\omega$ to be a small value and reduce it to zero in the final expressions.

The analysis shows that the oscillations of the longitudinal conductivity are determined by the integral $\int_{-\hbar \omega}^{0} d \varepsilon$ in Eq. (8), where the self-energy parts $X_{\varepsilon}$ and $X_{\varepsilon+\hbar \omega}$ have the opposite signs $19,20,21,22]$. The other regions of the integration over $\varepsilon$ in Eq. (8), $-\infty<\varepsilon<-\hbar \omega$ and $0<\varepsilon<\infty$, compensate the gauge term i $N e^{2} / m^{*} \omega$. To the first order in the parameter $\exp \left(-\pi / \omega_{c} \tau\right)$ the longitudinal conductivity is given by

$$
\sigma_{x x}=\frac{m^{*} \omega_{c}}{4 \pi^{2}} \sum_{n n^{\prime}} \frac{\left|\left(J_{x}\right)_{n n^{\prime}}\right|^{2}}{\left(E_{F}-E_{n}-X_{0+}\right)\left(E_{F}-E_{n^{\prime}}-X_{0-}\right)} .
$$

where $\left(J_{x}\right)_{n n^{\prime}}$ are the matrix elements of the current density operator, and $X_{0 \pm}$ are the limits of the self-energy parts at $\varepsilon \rightarrow 0, X_{0 \pm}=\lim _{\varepsilon \rightarrow 0 \pm} X_{\varepsilon}$. The oscillations of the Hall conductivity $\sigma_{x y}$ are determined by the whole range of the integration over $\varepsilon$ in Eq. (8), from $-\infty$ to $+\infty$. To the first order in the parameter $\exp \left(-\pi / \omega_{c} \tau\right)$ the Hall conductivity is derived to be

$$
\begin{gathered}
\sigma_{x y}=\frac{m^{*} \omega_{c}}{4 \pi^{2}} \sum_{n n^{\prime}}\left(J_{x}\right)_{n^{\prime} n}\left(J_{y}\right)_{n n^{\prime}} \\
\times\left[\frac{1}{\left(E_{F}-E_{n}-X_{0+}\right)\left(E_{F}-E_{n^{\prime}}-X_{0-}\right)}\right. \\
+2 \mathrm{i} \frac{\arctan \left[2 \tau\left(E_{F}-E_{n}\right) / \hbar\right]-\arctan \left[2 \tau\left(E_{F}-E_{n^{\prime}}\right) / \hbar\right]}{\left(E_{n}-E_{n^{\prime}}\right)^{2}} \\
\left.-\frac{E_{n^{\prime}}-E_{n}+\mathrm{i} \hbar / \tau}{\left(E_{n^{\prime}}-E_{n}\right)\left(E_{F}-E_{n}+\mathrm{i} \hbar / 2 \tau\right)\left(\mathrm{E}_{\mathrm{F}}-\mathrm{E}_{\mathrm{n}^{\prime}}-\mathrm{i} \hbar / 2 \tau\right)}\right] .
\end{gathered}
$$

Eqs. (9) and (10) describe the magnetoconductivity of the two-dimensional electron system in the regime of the Shubnikov-de Hass oscillations. The main contribution to the sums (910) is introduced by the terms with the energies $E_{n}, E_{n^{\prime}}$ close to the Fermi surface. Therefore on analytical calculation, when the summation over $n$ is replaced by an integration with the Poisson formula, the integration is performed over residua of the Green's

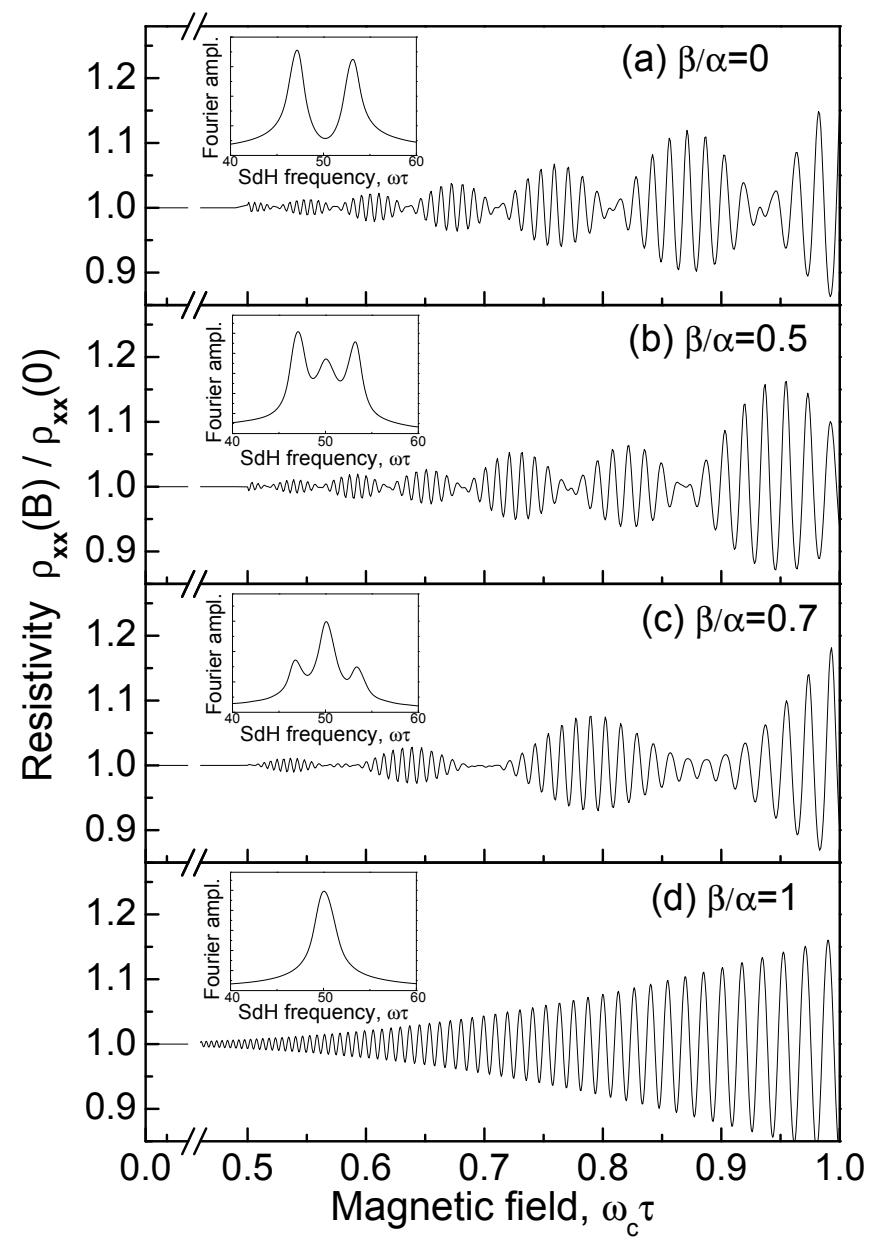

FIG. 2: The magnetic-field dependence of the resistivity $\rho_{x x}$ for different ratios of Rashba to Dresselhaus terms, $\beta / \alpha=$ $0,0.5,0.7,1$, at fixed Fermi energy $E_{F} \tau / \hbar=50$ and fixed Dresselhaus splitting $\alpha k_{F} \tau / \hbar=3$. The insets present the Fourier spectra of the corresponding dependencies $\rho_{x x}$ on $1 / \omega_{c} \tau$.

function. It allows one to avoid divergency at $n \rightarrow \infty$ in $\sigma_{x x}$ and $\sigma_{x y}$ that is caused by the increase of the matrix element $\left(J_{i}\right)_{n n^{\prime}}$ with $n$ [21, 22]. The similar summation "over residua" in numerical calculations can be performed substituting the matrix elements $\left(J_{i}\right)_{n n^{\prime}}$ by $\sqrt{2 E_{F} /\left(n \hbar \omega_{c}\right)}\left(J_{i}\right)_{n n^{\prime}}$.

\section{RESULTS AND DISCUSSION}

Figure 2 displays the magnetic-field dependence of the resistivity

$$
\rho_{x x}=\frac{\sigma_{x x}}{\sigma_{x x}^{2}+\sigma_{x y}^{2}}
$$

calculated numerically following Eqs. 9 10) for various ratios of Rashba to Dresselhaus spin-splitting constants, $\beta / \alpha$. The ratio $\beta / \alpha$ was varied modifying the strength 
of Rashba term, while the Dresselhaus term was kept constant. The insets in the Figures present the Fourier spectra of the corresponding dependencies $\rho_{x x}$ on $1 / \omega_{c} \tau$.

For the case of only one type of the $\boldsymbol{k}$-linear terms ( $\beta=0$, Fig. 2a) the Shubnikov-de Haas oscillations demonstrate the pronounced beats. The phase of the oscillations reverses at the node points. As shown in the inset, the spectrum of the oscillations consists of two harmonics, with the spectral positions corresponding to the energies of the spin subbands, $E_{F} \pm \alpha k_{F}$. The oscillating part of the resistivity is described analytically by $\exp \left(-\pi / \omega_{c} \tau\right) \cos \left(2 \pi \alpha k_{F} / \hbar \omega_{c}\right) \cos \left(2 \pi E_{F} / \hbar \omega_{c}\right)$.

In the presence of both Rashba and Dresselhaus contributions of comparable strengths the pattern of the magneto-oscillations modifies (Figs. 2b, 2k). As can be seen in the insets the central peak arises in the Fourier spectrum. For the parameter set presented in the Figure caption, the oscillations at $\beta / \alpha=0.5$ (Fig. 20) demonstrate the beat pattern similar by sight to that at $\beta=0$ (Fig. 2a), although the Fourier spectrum shows clearly the mixture of three harmonics. Further increase of the ratio $\beta / \alpha$ to 1 results in the noticeable modification of the magneto-oscillations (Fig. 2k). The beats become irregular, the reverse of the oscillation phase at nodes disappears, and with further approaching of $\beta / \alpha$ to 1 the nodes disappear.

In the case of the equal strengths of the Rashba and Dresselhaus terms, the beats vanish completely and the magneto-oscillations occur at a single frequency, although the $\boldsymbol{k}$-linear spin splitting remains for the most of the directions of the wave vector $\boldsymbol{k}$. In this particular case the oscillations are described by $\exp \left(-\pi / \omega_{c} \tau\right) \cos \left(2 \pi E_{F}^{\prime} / \hbar \omega\right)$, where $E_{F}^{\prime}=E_{F}+$ $2 m^{*} \alpha^{2} / \hbar^{2}$ is the Fermi level measured from the bottom of the spin subbands [14].

Such a behavior of the magneto-oscillations at various $\beta / \alpha$ can be understood qualitatively considering the simple semiclassical picture. In the framework of the semiclassical treatment the frequencies of the magnetooscillations correspond to the Borh-Sommerfeld quantization of an electron motion over the classical cyclotron orbits (Fig. 3). For the case when the spin-orbit splitting exceeds the cyclotron energy $\hbar \omega_{c}$, the electrons move over the cyclotron orbits, with the spins adiabatically oriented parallel or antiparallel to the effective magnetic field $\boldsymbol{B}_{\text {so }}$ induced by the spin-orbit coupling [13]. The orbits corresponding to the spins oriented along and opposite to $\boldsymbol{B}_{\text {so }}$ are split in $\boldsymbol{k}$-space. In the presence of the Dresselhaus term only (Fig. 3 ), the electron spectrum in zero external field has the form

$$
E_{\boldsymbol{k}, \pm}=\hbar^{2} k^{2} / 2 m^{*} \pm \alpha k
$$

Quantization of cyclotron orbits corresponding to the spins oriented parallel and antiparallel to $\boldsymbol{B}_{\text {so }}$ gives rise to two harmonics in the magneto-oscillations, i.e. the beating pattern.

In the presence of both Rashba and Dresselhaus contributions of comparable strengths (Fig. 3b) the electron
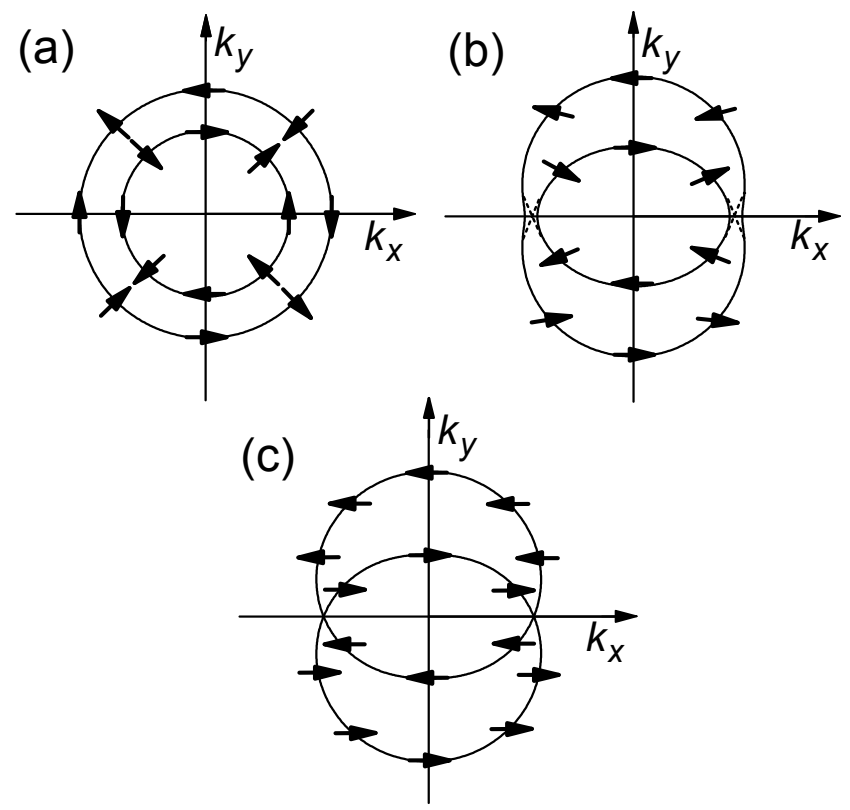

FIG. 3: Fermi contours for electrons in (001)-grown quantum wells in zero magnetic field in the presence of (a) Dresselhaus term only, $\beta=0$, (b) Rashba and Dresselhaus terms, (c) both contributions of the equal strengths, $\beta / \alpha=1$. Arrows indicate the orientation of spins. Dotted lines in Fig. (b) show possible transitions between the closely located orbits.

spectrum becomes strongly anisotropic [25]. The condition of adiabatic spin orientation along the cyclotron orbit is broken in certain points in the $\boldsymbol{k}$-space where the spin splitting is small. In the vicinity of the points the electron spin does not follow adiabatically the effective field $\boldsymbol{B}_{\text {so }}$, and electron transitions from one spin orbit to the other become possible. The magnetic breakdown occurs, i.e. the electron tunneling between the close cyclotron orbits 26]. The transitions corresponding to the magnetic breakdown between the orbits are shown in Fig. 3p with dotted lines. Quantization of the cyclotron motion with the transitions between the orbits results in appearance of the third (central) harmonics in the magneto-oscillations. The increase of the ratio $\beta / \alpha$ to 1 leads to increase of the probability of the tunneling between the orbits, i.e. to enhancement of the central harmonic and depression of the low- and high-frequency harmonics.

In the case of the equal strengths of the Rashba and Dresselhaus terms, the Fermi surface consists of two identical circles shifted relative to each other in the $\boldsymbol{k}$-space. For $\alpha=\beta$ the circles are shifted along $k_{y}$ and characterized by the spin states $| \pm 1 / 2\rangle$ onto the $x$-axis (Fig. 3 b). In this particular case electrons within each spin subband are quantized identically in the external magnetic field, and only one (central) harmonics remains in the spectrum of the magneto-oscillations. The magnetic breakdown between the subbands does not occur, because the electron spins in the subbands are opposite directed. The 
electron spin initially oriented parallel or antiparallel to $\boldsymbol{B}_{s o}$ keeps the orientation during the electron motion over the cyclotron orbit because the effective magnetic field is collinear to $x$-axis independent of the wave vector.

The situation when the Rashba contribution to the spin splitting is larger than the Dresselhaus term, $\beta / \alpha>$ 1 , is similar to the case of $\beta / \alpha<1$ considered above. Increase of the ratio $\beta / \alpha$ from 1 leads to appearance of the three harmonics in the spectrum of the magneto- oscillations again. When the Rashba contribution dominates, only two harmonics corresponding to the spinsplit subbands remain in the spectrum and the pattern of the magneto-oscillations is similar to that presented in Fig. 2].

This work was supported by the RFBR, the INTAS, programs of the RAS and the Russian Ministry of Science and Education, and Foundation "Dynasty" - ICFPM.
[1] Yu.A. Bychkov and É.I. Rashba, J. Phys. C: Solid State Phys., 17, 6039 (1984).

[2] M.I. D'yakonov and V.Yu. Kachorovskii, Fiz. Tech. Poluprovod. 20, 178 (1986) [Sov. Phys. Semicond. 20, 110 (1986)].

[3] J. Luo, H. Munekata, F.F. Fang, and P.J. Stiles, Phys. Rev. B 38, 10142 (1988); Phys. Rev. B 417685 (1990).

[4] Yu.L. Ivanov, P.S. Kop'ev, S.D. Suchalkin, and V.M. Ustinov, Pis'ma Zh. Eksp. Teor. Fiz. 53, 470 (1991) [JETP Lett. 53, 493 (1991)].

[5] B. Das, D.C. Miller, S. Datta, R. Reifenberger, W.P. Hong, P.K. Bhattacharya, J. Singh, and M. Jaffe, Phys. Rev. B 39, 1411 (1989); B. Das, S. Datta, and R. Reifenberger, Phys. Rev. B 418278 (1990).

[6] J. Nitta, T. Akazaki, H. Takayanagi, and T. Enoki, Phys. Rev. Lett. 78, 1335 (1997); C.-M. Hu, J. Nitta, T. Akazaki, H. Takayanagi, J. Osaka, P. Pfeffer, and W. Zawadzki, Phys. Rev. B 60, 7736 (1999).

[7] L.J. Cui, Y.P. Zeng, B.Q. Wang, Z.P. Zhu, L.Y. Lin, C.P. Jiang, S.L. Guo, and J.H. Chu, Appl. Phys. Lett. 80, 3132 (2002).

[8] G. Engels, J. Lange, Th. Schäpers, and H. Lüth, Phys. Rev. B 55, 1958 (1997); Th. Schäpers, G. Engels, J. Lange, Th. Klocke, M. Hollfelder, and H. Lüth, J. Appl. Phys. 83, 4324 (1998).

[9] P. Ramvall, B. Kowalski, and P. Omling, Phys. Rev. B 55, 7160 (1997).

[10] J.P. Heida, B.J. van Wees, J.J. Kuipers, T.M. Klapwijk, and G. Borghs, Phys. Rev. B 57, 11911 (1998).

[11] Ikai Lo, J.K. Tsai, W.J. Yao, P.C. Ho, L.-W. Tu, T.C. Chang, S. Elhamri, W.C. Mitchel, K.Y. Hsieh, J.H. Huang, and H.L. Huang, W.-C. Tsai, Phys. Rev. B 65, 161306 (2002).

[12] R.G. Mani, J.H. Smet, K. von Klitzing, V. Narayanamurti, W.B. Johnson, and V. Umansky, Phys. Rev. B 69, 193304 (2004).

[13] S. Keppeler and R. Winkler, Phys. Rev. Lett. 88, 046401 (2002).

[14] S.A. Tarasenko and N.S. Averkiev, Pis'ma Zh. Eksp.
Teor. Fiz. 75, 669 (2002) [JETP Lett. 75, 552 (2002)].

[15] U. Rössler and J. Kainz, Solid State Commun. 121, 313 (2002).

[16] B. Jusserand, D. Richards, G. Allan, C. Priester, and B. Etienne, Phys. Rev. B 51, 4707 (1995); D. Richards, B. Jusserand, G. Allan, C. Priester, and B. Etienne, Solid-State Electronics 40, 127 (1996).

[17] W. Knap, C. Skierbiszewski, A. Zduniak, E. LitwinStaszevska, D. Bertho, F. Kobbi, J.L. Robert, G.E. Pikus, F.G. Pikus, S.V. Iordanskii, V. Mosser, K. Zekenes, and Yu.B. Lyanda-Geller, Phys. Rev. B 53, 3912 (1996); J.B. Miller, D.M. Zumbühl, C.M. Marcus, Yu.B. Lyanda-Geller, D. Goldhaber-Gordon, K. Campman, and A.C. Gossard, Phys. Rev. Lett. 90, 076807 (2004).

[18] S.D. Ganichev, Petra Schneider, V.V. Bel'kov, E.L. Ivchenko, S.A. Tarasenko, W. Wegscheider, D. Weiss, D. Schuh, B.N. Murdin, P.J. Phillips, C.R. Pidgeon, D.G. Clarke, M. Merrick, P. Murzyn, E.V. Beregulin, and W. Prettl, Phys. Rev. B 68, 081302 (2003); S.D. Ganichev, V.V. Bel'kov, L.E. Golub, E.L. Ivchenko, Petra Schneider, S. Giglberger, J. Eroms, J. De Boeck, G. Borghs, W. Wegscheider, D. Weiss, and W. Prettl, Phys. Rev. Lett. 92, 256601 (2004).

[19] T. Ando, J. Phys. Soc. Jpn. 37, 1233 (1974).

[20] A. Isihara and L. Smrčka, J. Phys. C 19, 6777 (1986).

[21] N.S. Averkiev, L.E. Golub, S.A. Tarasenko, and M. Willander, J. Phys.: Condens. Matter 13, 2517 (2001).

[22] S.A. Tarasenko, Fiz. Tverd. Tela 44, 1690 (2002) [Phys. Solid State 44, 1769 (2002)].

[23] X.F. Wang and P. Vasilopoulos, Phys. Rev. B 67, 085313 (2003).

[24] M. Langenbuch, M. Suhrke, and U. Rössler, Phys. Rev. B 69, 125303 (2004).

[25] E.A. de Andrada e Silva, Phys. Rev. B 46, 1921 (1992).

[26] E.A. de Andrada e Silva, G.C. La Rocca, and F. Bassani, Phys. Rev. B 50, 8523 (1994). 